\title{
On the Separated Bumps Conjecture for Calderón-Zygmund Operators
}

\author{
Michael T. LACEY \\ (Received January 29, 2014; Revised March 25, 2014)
}

Abstract. Let $\sigma(d x)=\sigma(x) d x$ and $w(d x)=w(x) d x$ be two weights with nonnegative locally finite densities on $\mathbb{R}^{d}$, and let $1<p<\infty$. A sufficient condition for the norm estimate

$$
\int|T(\sigma f)|^{p} w(d x) \leq C_{T, \sigma, w}^{p} \int|f|^{p} \sigma(d x)
$$

valid for all Calderón-Zygmund operators $T$ is that the condition below holds.

$$
\sup _{Q \text { a cube }}\left\|\sigma^{1 / p^{\prime}}\right\|_{L} A_{(Q, d x /|Q|)} \varepsilon\left(\left\|\sigma^{1 / p^{\prime}}\right\|_{L} A_{(Q, d x /|Q|)} / \sigma(Q)^{1 / p^{\prime}}\right)\left[\frac{w(Q)}{|Q|}\right]^{1 / p}<\infty
$$

Here $A$ is Young function, with dual in the Pérez class $B_{p}$, and the function $\varepsilon(t)$ is increasing on $(1, \infty)$ with $\int^{\infty} \varepsilon(t)^{-p^{\prime}}(d t / t)<\infty$. Moreover, a dual condition holds, with the roles of the weights and $L^{p}$ indices reversed also holds. This is an alternate version of a result of Nazarov, Reznikov and Volberg $(p=2)$, one with a simpler formulation, and proof based upon stopping times.

Key words: weighted inequality, $A_{p}$, bumps, Orlicz spaces.

\section{Introduction}

Our subject is the two weight theory for Calderón-Zygmund operators. To say that $\sigma$ is a weight on $\mathbb{R}^{d}$ means that it is a locally finite, nonnegative, Borel measure. Throughout, we further assume that $\sigma$ is absolutely continuous with respect to Lebesgue measure, $\sigma(d x)=\sigma(x) d x$, and we will not distinguish the density from the weight. Fix $1<p<\infty$. For which pairs of weights $w$ and $\sigma$ does the following hold for all Calderón-Zygmund operators $T$ ?

2000 Mathematics Subject Classification : Primary 42B20; Secondary 42B25, 42B35.

Research supported in part by grant NSF-DMS 1265570 and the Australian Research Council through grant ARC-DP120100399. 


$$
\int|T(\sigma f)|^{p} w(d x) \leq C_{T, \sigma, w}^{p} \int|f|^{p} \sigma(d x)
$$

The precise definition for these and other terms will be given in the next section. Below, we will write $T_{\sigma} f:=T(\sigma f)$. In the case that $w(x)>0$ a.e., and $\sigma(x)=w(x)^{1-p^{\prime}}$, so that $w(x)^{1 / p} \sigma(x)^{1 / p^{\prime}} \equiv 1$, where $1=1 / p+1 / p^{\prime}$, the necessary and sufficient condition is the famous Muckenhoupt condition,

$$
[w]_{A_{p}}:=\sup _{Q} \frac{w(Q)}{|Q|}\left[\frac{\sigma(Q)}{|Q|}\right]^{p-1}<\infty .
$$

In the classical case of just the Hilbert transform, this is the result of HuntMuckenhoupt-Wheeden [9]. Thoroughly modern treatments of the $A_{p}$ theory are given in [11], [10], [15]. But, the two weight problem, in which $\sigma$ and $w$ have no explicit relationship, is far more complicated, as was recognized even at early stages of the subject.

The question addressed here is: What is the sharpest condition, expressible in terms similar to the $A_{p}$ condition, which is sufficient for the family of inequalities (1.1)? There are many results in the literature which modify (1.2) by requiring slightly higher integrability conditions on the densities of the weights. The sharpest of these conditions is the 'separated bump conjecture,' which arises from work of Cruz-Uribe and Pérez [7], [6], and Cruz-Uribe and Reznikov and Volberg [8].

To set the stage for this condition, observe that the $A_{p}$ condition can be rewritten as the $p$ th power of

$$
\sup _{Q}\left\|w^{1 / p}\right\|_{L^{p}(Q, d x /|Q|)} \cdot\left\|\sigma^{1 / p^{\prime}}\right\|_{L^{p^{\prime}}(Q, d x /|Q|)} .
$$

The bump conditions, in the two weight setting, strengthen this condition by replacing the $L^{p}$ norms by stronger ones. The strengthening is on the scale of Orlicz spaces. Set $\langle f\rangle_{Q}=|Q|^{-1} \int_{Q} f d x$, which is just the average of $f$. Write

$$
\langle f\rangle_{A, Q}:=\|f\|_{L^{A}(Q, d x /|Q|)},
$$

where $A$ is a Young function $A:[0, \infty) \rightarrow[0, \infty)$, and $L^{A}$ denotes the Luxembourg norm associated to $A$, and we have normalized Lebesgue measure on $Q$. The dual Young function to $A$ is denoted by $\bar{A}$. 
We write $A \in B_{p}$ if

$$
\int^{\infty} \frac{A(t)}{t^{p}} \cdot \frac{d t}{t}<\infty .
$$

A typical example of function $A(t)$ is just a bit smaller than $t^{p}$, for example $t^{p}(\log t)^{-1-\epsilon}$, for $\epsilon>0$, in which case $\langle f\rangle_{A, Q}$ is just a bit smaller than $\left\langle|f|^{p}\right\rangle_{Q}^{1 / p}$. To put the main conjecture in context, we recall the result of Carlos Pérez which is basic for us.

Theorem A (C. Pérez [19]) Suppose that $A \in B_{p}$. Then, there holds

$$
\left\|\sup _{Q \text { a cube }} \mathbf{1}_{Q}\langle f\rangle_{A, Q}\right\|_{p} \lesssim\|f\|_{p} .
$$

Moreover, the condition $A \in B_{p}$ is necessary for the inequality above.

This has the following implication for weighted inequalities. The Theorem below from [19] gives the sharpest ' $A_{p}$-like' sufficient conditions for the boundedness of the maximal function.

Theorem B (C. Pérez [19]) Suppose that $1<p<\infty$, and $A \in B_{p}$. Assume the condition below involving the dual function $\bar{A}$,

$$
[\sigma, w]_{\bar{A}, p^{\prime}}:=\sup _{Q \text { a cube }}\left\langle\sigma^{1 / p^{\prime}}\right\rangle_{\bar{A}, Q}\langle w\rangle_{Q}^{1 / p}<\infty
$$

Then this maximal function inequality holds: $\left\|M_{\sigma} f\right\|_{L^{p}(w)} \lesssim\|f\|_{L^{p}(\sigma)}$, where

$$
M_{\sigma} f=\sup _{Q} \mathbf{1}_{Q}\langle|f| \cdot \sigma\rangle_{Q}
$$

Since $A \in B_{p}, A$ must be a bit smaller than $t^{p}$, hence $\bar{A}$ must be a bit bigger than $t^{p^{\prime}}$. That is we will have $\left\|\sigma^{1 / p^{\prime}}\right\|_{\bar{A}, Q} \gtrsim\langle\sigma\rangle_{Q}^{1 / p^{\prime}}$, whence the condition (1.5) is stronger than the two weight $A_{p}$ condition. (It is known that the two weight $A_{p}$ condition only characterizes the weak-type norm of the maximal function.)

This Theorem answers a question of Cruz-Uribe and Pérez [6]. It was proved almost at the same time by Nazarov, Reznikov and Volberg [16] $(p=2)$, and by Lerner [15] for all $p$. 
Theorem $\mathbf{C}$ Let $\sigma$ and $w$ be two weights with densities, and $1<p<\infty$. Let $A \in B_{p}$ and $B \in B_{p^{\prime}}$. For all Calderón-Zygmund operators $T$, there holds

$$
\left\|T_{\sigma}: L^{p}(\sigma) \rightarrow L^{p}(w)\right\| \lesssim C_{T}\left\{\sup _{Q}\left\langle\sigma^{1 / p^{\prime}}\right\rangle_{\bar{A}, Q}\left\langle w^{1 / p}\right\rangle_{\bar{B}, Q}\right\} .
$$

In the inequality above, the bumps occur in the same product. The logic of the two weight theory suggests that the bumps could appear individually. Cruz-Uribe and Reznikov and Volberg [8] identified this conjecture as:

Conjecture 1.1 Let $\sigma$ and $w$ be two weights with densities, and $1<p<$ $\infty$. Let $A \in B_{p}$ and $B \in B_{p^{\prime}}$. For all Calderón-Zygmund operators $T$, there holds

$$
\left\|T_{\sigma}: L^{p}(\sigma) \rightarrow L^{p}(w)\right\| \lesssim C_{T}\left\{[\sigma, w]_{\bar{A}, p^{\prime}}+[w, \sigma]_{\bar{B}, p}\right\}\|f\|_{L^{p}(\sigma)} .
$$

The constant $C_{T}$ is defined in (2.1), and the implied constant depends upon the choice of $A, B$.

This has been difficult to verify. The very interesting paper of Nazarov, Reznikov and Volberg [17] proves a certain version of the conjecture in the case of $p=2$, under an additional condition which they speculate is necessary. The result below holds in all $L^{p}$, has fewer technical hypotheses, and contains [17].

Theorem 1.2 Let $\sigma$ and $w$ be two weights with densities, and $1<p<$ $\infty$. Let $A \in B_{p}$ and $B \in B_{p^{\prime}}$, and let $\varepsilon_{p}, \varepsilon_{p^{\prime}}$ be two monotonic increasing functions on $(1, \infty)$ which satisfy $\int_{1}^{\infty} \varepsilon_{p}(t)^{-p^{\prime}}(d t / t)=1$, and similarly for $\varepsilon_{p^{\prime}}$. Define

$$
\lceil\sigma, w\rceil_{\bar{A}, \varepsilon_{p}, p^{\prime}}:=\sup _{Q \text { a cube }} \varepsilon_{p}\left(1+\frac{\left\langle\sigma^{1 / p^{\prime}}\right\rangle_{\bar{A}, Q}}{\langle\sigma\rangle_{Q}^{1 / p^{\prime}}}\right)\left\langle\sigma^{1 / p^{\prime}}\right\rangle_{\bar{A}, Q}\langle w\rangle_{Q}^{1 / p} .
$$

For any Calderón-Zygmund operator, there holds

$$
\left\|T_{\sigma}: L^{p}(\sigma) \rightarrow L^{p}(w)\right\| \lesssim C_{T}\left\{\lceil\sigma, w\rceil_{\bar{A}, \varepsilon_{p}, p^{\prime}}+\lceil w, \sigma\rceil_{\bar{B}, \varepsilon_{p^{\prime}}, p}\right\}\|f\|_{L^{p}(\sigma)} .
$$

The constant $C_{T}$ is defined in (2.1). 
Compared to the condition in (1.7), the bound above penalizes the product $\left\langle\sigma^{1 / p^{\prime}}\right\rangle_{\bar{A}, Q}\langle w\rangle_{Q}^{1 / p}$ by the additional term involving $\varepsilon_{p}$. Now, $\varepsilon_{p}(t)$ has to satisfy an integrability condition that requires it to be, for example, of the order of

$$
\varepsilon(t) \simeq(\log t)^{+1 / p^{\prime}}(\log \log t)^{+1 / p^{\prime}}(\log \log \log t)^{+(1+\eta) / p^{\prime}}, \quad t \rightarrow \infty, \eta>0 .
$$

We prefer this formulation of the theorem, since it requires fewer hypotheses than [17]. In addition, the extra hypothesis involving $\varepsilon_{p}$ and $\varepsilon_{p^{\prime}}$ enter the proof in transparent, and seemingly unavoidable, way, see Lemma 4.5. Our approach can provide further insights into how to build counterexamples to Conjecture 1.1, in light of the fact that the Bellman approach followed in [8], is not 'reversible' in the separated bump setting,

The study of 'bumped' $A_{p}$ conditions have an extensive history within just the subject of weighted estimates for singular integrals. Related conditions have arisen in the spectral theory of Schrödinger operators [2], and in the study of Sarason's conjecture on the composition of Toeplitz operators [21], [25]. The article of Neugebauer [18] firmly introduced the subject into weighted estimates, and Pérez [19], [20] promoted their fine study. The reader is referred to [8], [24], [3], [4], [5] for more history on this subject.

More recent developments have been as follows. The paper [8] raised as a conjecture the Theorem proved above, and established variants of the result where the $A(t)$ were ' $\log$ bumps', namely $A(t) \simeq t^{p}(\log (\mathrm{e}+t))^{-1-\delta}$, for $\delta>0$. The papers of Lerner [15, Theorem 1.5] and Nazarov, Reznikov \& Volberg [16] $(p=2)$ established Theorem C. The latter estimate is strictly stronger than (1.6), as an example of [1, Section 7] shows. Variants of these results were proved in the setting of spaces of homogeneous type in [1].

The next section collects standard aspects of the subject, and can be referred back to as needed. The proof of the main theorem is in Section 4 .

\section{Notation, Background}

Constants are suppressed: $\mathrm{By} A \lesssim B$, it is meant that there is an absolute constant $c$ so that $A \leq c B$.

We say that $K: \mathbb{R}^{d} \times \mathbb{R}^{d} \rightarrow \mathbb{R}$ is a Calderón-Zygmund kernel if for some constants $\Lambda>0$, and $C_{K}>0$, and $0<\eta<1$, such that these conditions hold: For $x, x^{\prime}, y \in \mathbb{R}^{d}$ 


$$
\begin{gathered}
\|K(\cdot, \cdot)\|_{\infty}<\infty, \quad K(x, y)=0, \quad|x-y|>\Lambda, \\
|K(x, y)|<C_{K}|x-y|^{-d}, \quad x \neq y, \\
\left|K(x, y)-K\left(x^{\prime}, y\right)\right|<C_{K} \frac{\left|x-x^{\prime}\right|^{\eta}}{|x-y|^{d+\eta}}, \quad \text { if } 2\left|x-x^{\prime}\right|<|x-y|,
\end{gathered}
$$

and a fourth condition, with the roles of the first and second coordinates of $K(x, y)$ reversed also holds. These are typical conditions, although in the first condition, we have effectively truncated the kernel, at the diagonal and infinity. The effect of this is that we needn't be concerned with principal values.

Given a Calderón-Zygmund kernel $K$ as above, we can define

$$
T f(x):=\int K(x, y) f(y) d y
$$

which is defined for all $f \in L^{2}$ and $x \in \mathbb{R}^{d}$. We say that $T$ is a CalderónZygmund operator, since it necessarily extends to a bounded operator on $L^{2}\left(\mathbb{R}^{d}\right)$. We define

$$
C_{T}:=C_{K}+\left\|T: L^{2} \rightarrow L^{2}\right\|
$$

It is well-known that $T$ is also bounded on $L^{p}, 1<p<\infty$, with norm controlled by $C_{T}$.

To say that $A:[0, \infty) \rightarrow[0, \infty)$ is a Young function means that $A(0)=$ $0, A(1)=1, A$ is convex, continuous increasing and $A(t) / t \rightarrow \infty$, as $t \rightarrow \infty$. The Luxembourg norm associated to $A$, on a measure space $(X, \mu)$ is given by

$$
\|f\|_{L^{A}(X, \mu)}:=\inf \left\{\lambda>0: \int_{X} A(|f| / \lambda) d \mu \leq 1\right\} .
$$

The infimum of the empty set is taken to be $\infty$.

We most frequently work with Young functions of the form $A(x)$ of the form $c t^{p} \alpha(t)$, where $1<p<\infty$, and $\alpha(t)$ is constant for $0 \leq t \leq 1$, and $\alpha(t)$ is slowly varying at infinity, thus $\alpha(2 t) / \alpha(t) \rightarrow 1$ as $t \rightarrow \infty$. The dual to $A$ is defined by $\bar{A}(t) \simeq \int_{0}^{t}\left(A^{\prime}\right)^{-1}(s) d s$. In this case, note that 


$$
\bar{A}(t)=\int_{0}^{t}\left[\frac{s}{\alpha(s)}\right]^{1 /(p-1)} d s \simeq t^{p^{\prime}} \alpha(t)^{-1 /(p-1)} .
$$

This is also a Young function, and we will use the generalized Hölder's inequality, in the form below, but note $X$ must be a probability space.

$$
\int_{X} f \cdot g d \mu \lesssim\|f\|_{L^{A}(X, \mu)}\|g\|_{L^{\bar{A}}(X, \mu)}, \quad \mu(X)=1 .
$$

\section{General Considerations}

We use the following consequence of Lerner's remarkable median inequality. We say that $T$ is a sparse operator if $T f=\sum_{Q \in \mathcal{Q}}\langle f\rangle_{Q} \mathbf{1}_{Q}$, where $\mathcal{Q}$ is a collection of dyadic cubes for which

$$
\left|\bigcup_{Q^{\prime} \in \mathcal{Q}: Q^{\prime} \subsetneq Q} Q^{\prime}\right| \leq \frac{1}{2}
$$

We will also refer to $\mathcal{Q}$ as sparse, and will typically suppress the dependence of $T$ on $\mathcal{Q}$. Trivially, any subset of a sparse collection is sparse. (The definition of sparseness in [17] is a bit more general, but this is not needed herein.)

A sparse operator is bounded on all $L^{p}$, and in fact, is a 'positive dyadic Calderón-Zygmund operator.' And the class is sufficiently rich to capture the norm behavior of an arbitrary Calderón-Zygmund operator, which is the wonderful observation of Andrei Lerner:

Theorem D ([14, Theorem 1.1]) We have the equivalence

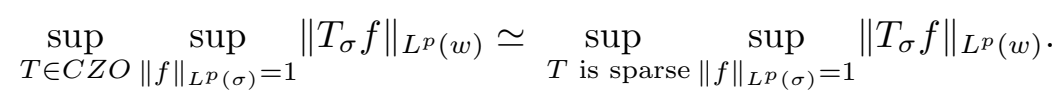

We need this consequence of the two weight theory of E. Sawyer [23]. A detailed study of the dyadic setting is in [12], and an efficient proof is given at the end of [11].

Theorem $\mathbf{E}([23]) \quad$ For a sparse operator $T$ with sparse collection $\mathcal{Q}$, the norm is approximately

$$
\left\|T_{\sigma}: L^{p}(\sigma) \rightarrow L^{p}(w)\right\| \simeq N_{\mathcal{Q}}
$$


where $N_{\mathcal{Q}}$ is the best constant $N$ in the inequalities

$$
\begin{array}{cc}
\int_{Q_{0}}\left|\sum_{Q \in \mathcal{Q}: Q \subset Q_{0}}\langle\sigma\rangle_{Q} \mathbf{1}_{Q}\right|^{p} d w \lesssim N_{\mathcal{Q}}^{p} \sigma\left(Q_{0}\right), \quad Q_{0} \in \mathcal{Q}, \\
\int_{Q_{0}}\left|\sum_{Q \in \mathcal{Q}: Q \subset Q_{0}}\langle w\rangle_{Q} \mathbf{1}_{Q}\right|^{p^{\prime}} d \sigma \lesssim N_{\mathcal{Q}}^{p^{\prime}} w\left(Q_{0}\right), \quad Q_{0} \in \mathcal{Q} .
\end{array}
$$

\section{Proof of Main Theorem}

We consider the first group of testing inequalities in (3.2), and the proof given will, by duality, apply to the second testing inequalities. Assume for the remainder of the argument that $Q_{0}$ is a fixed cube, $\mathcal{Q}$ is a sparse collection of cubes contained in $Q_{0}, \mathcal{Q}$ contains $Q_{0}$. In fact, we strengthen the sparseness condition (3.1) to

$$
\left|\bigcup_{Q^{\prime} \in \mathcal{Q}: Q^{\prime} \subsetneq Q} Q^{\prime}\right|<10^{-p}|Q|, \quad Q \in \mathcal{Q} .
$$

This can be accomplished by dividing $\mathcal{Q}$ meeting the condition (3.1) into a bounded number (as a function of $1<p<\infty$ ) of subcollections. Set

$$
\rho(Q):=\frac{\left\langle\sigma^{1 / p^{\prime}}\right\rangle_{\bar{A}, Q}}{\langle\sigma\rangle_{Q}^{1 / p^{\prime}}} \geq 1
$$

These are the ratios that appear in the argument of $\varepsilon_{p}(t)$. In addition, we are free to assume that $\varepsilon_{p}(t)$ does not grow faster than a power of $\log t$ at infinity.

Assume that for all $Q \in \mathcal{Q}$,

$$
\varepsilon_{p}(\rho(Q))\left\langle\sigma^{1 / p^{\prime}}\right\rangle_{\bar{A}, Q}\langle w\rangle_{Q}^{1 / p} \simeq \varepsilon_{p}\left(\rho\left(Q_{0}\right)\right)\left\langle\sigma^{1 / p^{\prime}}\right\rangle_{\bar{A}, Q_{0}}\langle w\rangle_{Q_{0}}^{1 / p}:=\alpha
$$

We will show that

$$
\int_{Q_{0}}\left|\sum_{Q \in \mathcal{Q}}\langle\sigma\rangle_{Q} \mathbf{1}_{Q}\right|^{p} d w \lesssim \alpha^{p} \sigma\left(Q_{0}\right) .
$$


This is enough to conclude the first of the two testing inequalities in (3.2), by a trivial summation of $\alpha$ over a geometrically decreasing sequence of values.

We invoke a version of the parallel corona, originating in [13], also see the last page of [11]. The first step is proceed to (3.2) by duality. Thus, for $g$ a non-negative function in $L^{p^{\prime}}\left(Q_{0}, w\right)$, we will bound the form

$$
\sum_{Q \in \mathcal{Q}}\langle\sigma\rangle_{Q}\langle w\rangle_{Q}\langle g\rangle_{Q}^{w} \cdot|Q| \lesssim \alpha \sigma\left(Q_{0}\right)^{1 / p}\|g\|_{L^{p^{\prime}}(w)}
$$

Here $\langle g\rangle_{Q}^{w}=w(Q)^{-1} \int_{Q} g w(d x)$.

Construct stopping data for $g$ as follows. For any cube $Q \in \mathcal{Q}$, set $\operatorname{ch}_{\mathcal{T}}(Q)$ to be the largest subcubes $Q^{\prime} \in \mathcal{Q}$, contained in $Q$ such that $\langle g\rangle_{Q^{\prime}}^{w}>$ $10\langle g\rangle_{Q}^{w}$. Then, set $\mathcal{T}:=\bigcup_{k=0}^{\infty} \mathcal{T}_{k}$ where $\mathcal{T}_{0}:=\left\{Q_{0}\right\}$, and inductively $\mathcal{T}_{k+1}:=$ $\bigcup_{Q \in \mathcal{T}_{k}} \operatorname{ch}_{\mathcal{T}}(Q)$. Define for $T \in \mathcal{T}$

$$
T_{\sharp}:=T \backslash \bigcup_{T^{\prime} \in \operatorname{ch}_{\mathcal{T}}(T)} T^{\prime}
$$

It follows that $w\left(T_{\sharp}\right) \geq(1 / 2) w(T)$. Define

$$
g_{T}=\langle g\rangle_{T}^{w} \mathbf{1}_{T_{\sharp}}+\sum_{T^{\prime} \in \operatorname{ch}_{\mathcal{T}}(T)}\langle g\rangle_{T^{\prime}}^{w} \mathbf{1}_{T^{\prime}} .
$$

Let $Q^{t}$ be the minimal element of $\mathcal{T}$ that contains $Q$. Observe that if $Q^{t}=T$, then $\langle g\rangle_{Q}^{w}=\left\langle g_{T}\right\rangle_{Q}^{w}$. Moreover, by the maximal function estimate

$$
\sum_{T \in \mathcal{T}}\left(\langle g\rangle_{T}^{w}\right)^{p^{\prime}} w(T) \lesssim\|g\|_{L^{p^{\prime}(w)}}^{p^{\prime}}
$$

The construction of stopping data for $\sigma$ is not dual. For any $Q \in \mathcal{Q}$, set $\operatorname{ch}_{\mathcal{S}}(Q)$ to the largest subcubes $Q^{\prime} \in \mathcal{Q}$, contained in $Q$ such that $\langle\sigma\rangle_{Q^{\prime}}>$ $10\langle\sigma\rangle_{Q}$ or $Q^{\prime} \in \mathcal{T}$. (We restart the stopping cubes at each member of $\mathcal{T}$ !) Then, set $\mathcal{S}:=\bigcup_{k=0}^{\infty} \mathcal{S}_{k}$ where $\mathcal{S}_{0}:=\left\{Q_{0}\right\}$, and inductively $\mathcal{S}_{k+1}:=$ $\bigcup_{Q \in \mathcal{S}_{k}} \operatorname{ch}_{\mathcal{S}}(Q)$. Let $Q^{s}$ be the minimal element of $\mathcal{S}$ that contains $Q$.

This is then the basic estimate.

Lemma 4.1 There holds, uniformly in $T \in \mathcal{T}$, 


$$
\sum_{Q: Q^{t}=T}\langle\sigma\rangle_{Q}\langle w\rangle_{Q} \cdot|Q| \lesssim \alpha\left[\sum_{Q: Q^{t}=T}\left\langle\sigma^{1 / p}\right\rangle_{A, Q}^{p}|Q|\right]^{1 / p} w(T)^{1 / p^{\prime}}
$$

Proof of (4.5). Denoting $Q^{*}:=\left(Q^{s}, Q^{t}\right)$, observe that the left side of (4.5) is

$$
\sum_{S \in \mathcal{S}} \sum_{T \in \mathcal{T}} \sum_{Q: Q^{*}=(S, T)}\langle\sigma\rangle_{Q}\langle w\rangle_{Q}\langle g\rangle_{Q}^{w} \cdot|Q|
$$

The average $\langle g\rangle_{Q}^{w}=\left\langle g_{T}\right\rangle_{Q}^{w}$. We must have $S \subset T$ since $\mathcal{T} \subset \mathcal{S}$, but also we can restrict to $S^{t}=T$, for otherwise $Q^{t} \subsetneq T$. Therefore, the sum above is dominated by

$$
\begin{aligned}
(4.10) & =\sum_{T \in \mathcal{T}}\left\langle g_{T}\right\rangle_{Q}^{w} \sum_{Q: Q^{t}=T}\langle\sigma\rangle_{Q}\langle w\rangle_{Q} \cdot|Q| \\
& \lesssim \alpha \sum_{T \in \mathcal{T}}\left\langle g_{T}\right\rangle_{Q}^{w}\left[\sum_{Q: Q^{t}=T}\left\langle\sigma^{1 / p}\right\rangle_{A, Q}^{p}|Q|\right]^{1 / p} w(T)^{1 / p^{\prime}}
\end{aligned}
$$

Here, of course, we have applied (4.9). Apply Hölder's inequality. We have by sparseness and Pérez' maximal function estimate (1.4),

$$
\sum_{Q \in \mathcal{Q}}\left\langle\sigma^{1 / p}\right\rangle_{A, Q}^{p}|Q| \lesssim \int_{Q_{0}} \sup _{Q}\left\langle\sigma^{1 / p}\right\rangle_{A, Q}^{p} \mathbf{1}_{Q} d x \lesssim \sigma\left(Q_{0}\right)
$$

(Recall that $\mathcal{T} \subset \mathcal{S}$.) And, on the other, the estimate (4.8).

The obstacle to an easy proof of (4.9) is that the cubes $Q \in \mathcal{Q}$ need not be $w$-Carleson. We give the proof in three steps, in which we sum only over certain cubes; the first two are very easy. Write $\mathcal{Q}_{0}=\left\{Q_{0}\right\}$, and inductively set $\mathcal{Q}_{k+1}$ to be the maximal elements $Q \in \mathcal{Q}$ which are strictly contained in some $Q^{\prime} \in \mathcal{Q}_{k}$. The basic fact, a consequence of (4.1), is that for all $k$, $Q \in \mathcal{Q}_{k}$,

$$
\sum_{\substack{Q^{\prime} \in \mathcal{Q}_{k+j} \\ Q^{\prime} \subset Q}}\left|Q^{\prime}\right| \lesssim 10^{-p j}|Q|, \quad j \in \mathbb{N}
$$

We write $i_{T}(Q)=k$ if for some integer $\ell, T \in \mathcal{Q}_{\ell}$ and $Q \subset T$ with 
$Q \in \mathcal{Q}_{\ell+k}$. Our first easy estimate is as follows: We require that the average value of $w$ is not too large in the sum below.

Lemma 4.2 There holds, uniformly in $T \in \mathcal{T}$,

$$
\sum_{\substack{Q: Q^{t}=T \\\langle w\rangle_{Q}^{1 / p}<2^{2 i} T^{(Q)}\langle w\rangle_{T}^{1 / p}}}\langle\sigma\rangle_{Q}\langle w\rangle_{Q} \cdot|Q| \lesssim \alpha\left[\sum_{Q: Q^{t}=T}\left\langle\sigma^{1 / p}\right\rangle_{A, Q}^{p}|Q|\right]^{1 / p} w(T)^{1 / p^{\prime}}
$$

Proof. The restriction on $w$ in the sum in (4.12), with the estimate (4.11), immediately imply that

$$
\sum_{\substack{Q: Q^{t}=T \\\langle w\rangle_{Q}^{1 / p}<2^{2 i} T^{(Q)}\langle w\rangle_{T}^{1 / p}}} w(Q) \lesssim w(T) .
$$

Use the inequality below, a consequence of the generalized Hölder's inequality (2.3),

$$
\begin{aligned}
\langle\sigma\rangle_{Q}\langle w\rangle_{Q} & \lesssim\left\langle\sigma^{1 / p}\right\rangle_{A, Q}\left\{\left\langle\sigma^{1 / p^{\prime}}\right\rangle_{\bar{A}, Q}\langle w\rangle_{Q}^{1 / p}\right\}\langle w\rangle_{Q}^{1 / p^{\prime}} \\
& \lesssim \alpha\left\langle\sigma^{1 / p}\right\rangle_{A, Q}\langle w\rangle_{Q}^{1 / p^{\prime}}
\end{aligned}
$$

(We ignore the term $\varepsilon_{p}(\rho(Q))$ here.) Thus, we need only bound the restricted sum over $Q$ of the terms

$$
\left\langle\sigma^{1 / p}\right\rangle_{A, Q}|Q|^{1 / p} w(Q)^{1 / p^{\prime}}
$$

It is clear that Hölder's inequality completes the proof.

In our second easy estimate, we impose the restriction that the average of $\sigma$ has become rather small.

Lemma 4.3 There holds, uniformly in $T \in \mathcal{T}$,

$$
\begin{aligned}
& \sum_{S \in \mathcal{S}: S^{t}=T} \sum_{\substack{Q: Q^{t}=S \\
\langle\sigma\rangle^{1 / p}<2^{-i_{S}(Q) / 2}\langle\sigma\rangle^{1 / p}}}\langle\sigma\rangle_{Q}\langle w\rangle_{Q} \cdot|Q| \\
& \langle\sigma\rangle_{Q}^{1 / p}<2^{-i_{S}(Q) / 2}\langle\sigma\rangle_{S}^{1 / p}
\end{aligned}
$$




$$
\lesssim \alpha\left[\sum_{S \in \mathcal{S}: S^{t}=T}\left\langle\sigma^{1 / p}\right\rangle_{A, Q}^{p}|Q|\right]^{1 / p} w(T)^{1 / p^{\prime}}
$$

Proof. The restriction on $\sigma$ in the sum in (4.14) immediately imply that for each $S$

$$
\begin{aligned}
& \sum_{\substack{Q: Q^{s}=S \\
<2^{-i_{S}(Q) / 2}\langle\sigma\rangle_{S}^{1 / p}}}\langle\sigma\rangle_{Q} \mathbf{1}_{Q} \lesssim\langle\sigma\rangle_{S} \mathbf{1}_{S} . \\
& \langle\sigma\rangle_{Q}^{1 / p}<2^{-i_{S}(Q) / 2}\langle\sigma\rangle_{S}^{1 / p}
\end{aligned}
$$

Then, estimate

$$
\begin{aligned}
\operatorname{LHS}(4.14) & =\int_{T} \sum_{S \in \mathcal{S}: S^{t}=T} \sum_{\substack{Q: Q^{s}=S \\
\langle\sigma\rangle_{Q}^{1 / p}<2^{-i} S^{(Q) / 2}\langle\sigma\rangle_{S}^{1 / p}}}\langle\sigma\rangle_{Q} \mathbf{1}_{Q} d w \\
& \lesssim \int_{T} \sum_{S \in \mathcal{S}: S^{t}=T}\langle\sigma\rangle_{S} \mathbf{1}_{S} d w \\
& \lesssim w(T)^{1 / p^{\prime}}\left[\left.\int_{T} \sum_{S \in \mathcal{S}: S^{t}=T}\langle\sigma\rangle_{S} \mathbf{1}_{S}\right|^{p} d w\right]^{1 / p} \\
& \lesssim w(T)^{1 / p^{\prime}}\left[\int_{T} \sum_{S \in \mathcal{S}: S^{t}=T}\langle\sigma\rangle_{S}^{p} \mathbf{1}_{S} d w\right]^{1 / p}
\end{aligned}
$$

This just depends upon the fact that for each $x$, the sequence of numbers $\langle\sigma\rangle_{S} \mathbf{1}_{S}(x)$ is growing at least geometrically, so that we can pass the $p$ th power inside the sum.

Continuing, we note that as a consequence of (4.13),

$$
\langle\sigma\rangle_{S}^{p} w(S)=\langle\sigma\rangle_{S}^{p}\langle w\rangle_{S} \cdot|S| \lesssim \alpha^{p}\left\langle\sigma^{1 / p}\right\rangle_{A, S}^{p}|S|
$$

And this completes the proof. (Again, the role of $\varepsilon_{p}$ is ignored.)

Remark 4.4 The argument above, pushing the $p$ th power inside the sum in (4.15) is key. It for instance encapsulates the proof of Theorem B: Combine (1.4) with Sawyer's two weight maximal function theorem [22], using the argument (4.15) to verify the testing condition. But, there is nothing quite so simple in the current setting - the sparse operators are just a bit 
bigger than the maximal function. Nevertheless, we want to appeal to an argument similar to (4.15) below. To do so, we will absolutely need a certain integrability condition on the function $\varepsilon_{p}(t)$.

The third, and decisive, sum is over cubes complementary to the previous two.

Lemma 4.5 There holds, uniformly in $T \in \mathcal{T}$,

$$
\sum_{S \in \mathcal{S}: S^{t}=T} \sum_{Q: Q^{s}=S}^{3}\langle\sigma\rangle_{Q}\langle w\rangle_{Q} \cdot|Q| \lesssim \alpha\left[\sum_{Q: Q^{t}=T}\left\langle\sigma^{1 / p}\right\rangle_{A, Q}|Q|\right]^{1 / p} w(T)^{1 / p}
$$

Above the superscript ${ }^{3}$ on the second sum means that we sum over those $Q$ such that

$$
\langle w\rangle_{Q}^{1 / p} \geq 2^{2 i_{T}(Q)}\langle w\rangle_{T}^{1 / p}, \quad \text { and } \quad\langle\sigma\rangle_{Q}^{1 / p} \geq 2^{-i_{S}(Q) / 2}\langle\sigma\rangle_{S}^{1 / p}
$$

Proof. The role of $\varepsilon_{p}$ is now key. The main point of the assumptions on the sum in this case is that the ratios $\rho(Q)$ decrease at least geometrically, holding the stopping parents fixed:

$$
\rho(Q) \lesssim 2^{-i_{T}(Q) / 2} \rho(T), \quad Q \in \mathcal{Q}, Q^{s}=S, Q^{t}=T .
$$

Indeed, exploiting the definitions (4.2), (4.3), and the conditions in (4.17),

$$
\begin{aligned}
\varepsilon_{p}(\rho(Q))^{-1} \alpha & \simeq\left\langle\sigma^{1 / p^{\prime}}\right\rangle_{\bar{A}, Q}\langle w\rangle_{Q}^{1 / p} \\
& \simeq \rho(Q)\langle\sigma\rangle_{Q}^{1 / p^{\prime}}\langle w\rangle_{Q}^{1 / p} \\
& \gtrsim 2^{-i_{S}(Q) / 2+2 i_{T}(Q)} \rho(Q)\langle\sigma\rangle_{S}^{1 / p^{\prime}}\langle w\rangle_{T}^{1 / p} \\
& \gtrsim 2^{i_{T}(Q)} \rho(Q)\langle\sigma\rangle_{S}^{1 / p^{\prime}}\langle w\rangle_{T}^{1 / p} \\
& \gtrsim 2^{i_{T}(Q)} \rho(Q)\langle\sigma\rangle_{T}^{1 / p^{\prime}}\langle w\rangle_{T}^{1 / p} \\
& \simeq 2^{i_{T}(Q)} \frac{\rho(Q)}{\rho(T)}\langle\sigma\rangle_{\bar{A}, T}^{1 / p^{\prime}}\langle w\rangle_{T}^{1 / p} \\
& \simeq \alpha 2^{i_{T}(Q)} \frac{\rho(Q)}{\rho(T)} \varepsilon_{p}(\rho(T))^{-1}
\end{aligned}
$$


Summarizing, there holds

$$
2^{-i_{T}(Q)} \gtrsim \frac{\rho(Q)}{\rho(T)} \frac{\varepsilon_{p}(\rho(Q))}{\varepsilon_{p}(\rho(T))} \gtrsim\left[\frac{\rho(Q)}{\rho(T)}\right]^{2},
$$

since we assume that $\varepsilon_{p}(t)$ grows more slowly than a power of log at infinity. This proves (4.18).

The integrability condition on the function $\varepsilon_{p}$ is used this way. By the monotonicity of $\varepsilon_{p}(t)$, the condition $\int_{1}^{\infty} \varepsilon(t)^{-p^{\prime}}(d t / t)=1$, and the geometric decay (4.18), we see that

$$
\sum_{S \in \mathcal{S}: S^{t}=T} \sum_{Q: Q^{s}=S}^{3} \varepsilon(\rho(Q))^{-p^{\prime}} \mathbf{1}_{Q}(x) \lesssim \mathbf{1}_{T}(x) .
$$

We will rely upon this estimate below, which holds for all $Q \in \mathcal{Q}$.

$$
\begin{aligned}
\langle\sigma\rangle_{Q}^{p}\langle w\rangle_{Q} & \lesssim\left\langle\sigma^{1 / p}\right\rangle_{A, Q}^{p}\left\langle\sigma^{1 / p^{\prime}}\right\rangle_{\bar{A}_{, Q}}^{p}\langle w\rangle_{Q} \\
& \simeq \varepsilon_{p}(\rho(Q))^{-p}\left\langle\sigma^{1 / p}\right\rangle_{A, Q}^{p}\left\{\varepsilon_{p}(\rho(Q))^{p}\left\langle\sigma^{1 / p^{\prime}}\right\rangle_{\bar{A}_{, Q}}^{p}\langle w\rangle_{Q}\right\} \\
& \simeq \alpha^{p} \varepsilon(\rho(Q))^{-p}\left\langle\sigma^{1 / p}\right\rangle_{A, Q}^{p} .
\end{aligned}
$$

Now, the proof can be completed. We can estimate as below, inserting $\varepsilon(\rho(Q))$ to the zero power, and applying Hölder's inequality.

$$
\begin{aligned}
\operatorname{LHS}(4.16) & =\int_{T} \sum_{S \in \mathcal{S}: S^{t}=T} \sum_{Q: Q^{*}=(S, T)}^{3} \varepsilon(\rho(Q))^{-1+1}\langle\sigma\rangle_{Q} \mathbf{1}_{Q} d w \\
& \lesssim w(T)^{1 / p^{\prime}}\left[\int_{T} \sum_{Q: Q^{t}=T} \varepsilon(\rho(Q))^{p}\langle\sigma\rangle_{Q}^{p} \mathbf{1}_{Q} d w\right]^{1 / p} \\
& =w(T)^{1 / p^{\prime}}\left[\sum_{Q: Q^{t}=T} \varepsilon(\rho(Q))^{p}\langle\sigma\rangle_{Q}^{p}\langle w\rangle_{Q}|Q|\right]^{1 / p} \\
& \lesssim \alpha w(T)^{1 / p^{\prime}}\left[\sum_{Q: Q^{t}=T}\left\langle\sigma^{1 / p}\right\rangle_{A, Q}^{p}|Q|\right]^{1 / p}
\end{aligned}
$$

This completes the proof. 


\section{Log and LogLog Bumps}

We illustrate the implications of our main theorem for certain 'borderline' bumps. By a $\log$ bump, we mean function in $B_{p}$ of the form

$$
L_{p^{\prime}, \eta}(t) \simeq t^{p}\left(\log t^{p^{\prime}}\right)^{-1-(p-1) \eta}, \quad \eta>0, t \rightarrow \infty
$$

In this case, it follows from (2.2) that the dual Young function is $\overline{L_{p^{\prime}, \eta}}(t) \simeq$ $t^{p^{\prime}}(\log t)^{1 /(p-1)+\eta}$. Throughout this section, $\log t:=1+\max \{0, \log t\}$.

By a $\log$-log bump, we mean functions in $B_{p}$ of the form

$$
\begin{gathered}
\Lambda_{p^{\prime}, \eta}(t) \simeq t^{p}(\log t)^{-1}(\log t)^{-1-(p-1) \eta}, \quad \eta>0, t \rightarrow \infty . \\
\overline{\Lambda_{p^{\prime}, \eta}}(t) \simeq t^{p^{\prime}}(\log t)^{1 /(p-1)}(\log \log t)^{1 /(p-1)+\eta}
\end{gathered}
$$

We show that all the log bumps fall under the scope of our main theorem, and that log-log bumps do as well, provided the power on the bump is sufficiently large.

Proposition 5.1 These two assertions hold:

(1) Suppose that $\eta>0$, and that $\sigma$ and $w$ are a pair of weights such that $[\sigma, w]_{L_{p^{\prime}, \eta}, p^{\prime}}<\infty$, as defined in (1.5). Then, for $\varepsilon(t)=t^{\eta / 2 p^{\prime}}$, we have $\lceil\sigma, w\rceil \frac{L_{p^{\prime}, \eta / 2}}{\varepsilon_{p}, p^{\prime}}<\infty$, where the latter expression is defined in (1.8).

(2) Suppose that $\eta>0$, and that $\sigma$ and $w$ are a pair of weights such that $[\sigma, w]_{\overline{\Lambda_{p^{\prime}, 1+\eta}}, p^{\prime}}<\infty$. Then, set $\varepsilon_{p}(t)=(\log t)^{1 / p^{\prime}+\eta^{\prime} / p^{\prime}}$, we have $\lceil\sigma, w\rceil_{\Lambda_{p^{\prime}, \eta^{\prime} / p}, \varepsilon_{p}, p^{\prime}}<\infty$.

Moreover, in both cases,

$$
\int^{\infty} \varepsilon_{p}(t)^{-p^{\prime}} \frac{d t}{t}<\infty
$$

This relies upon the observations in [17], [14] concerning selfimprovement of Orlicz norms, and we will specialize their more general considerations. For the bump functions that we are interested in, write $\bar{A}(t)=t^{p^{\prime}} \beta\left(t^{p^{\prime}}\right)$, so that for $u$ sufficiently large,

$$
\beta(u) \simeq \begin{cases}(\log u)^{1 /(p-1)+\eta} & A=L_{p^{\prime}, \eta} \\ (\log u)^{1 /(p-1)}(\log \log u)^{1 /(p-1)+\eta} & A=\Lambda_{p^{\prime}, \eta}\end{cases}
$$


Also, for a cube $Q$, set

$$
D_{Q}(\lambda):=\frac{1}{|Q|}|\{x \in Q: \sigma(x)>\lambda\}| .
$$

Set $B(t)=t \beta(t)$. We have

Lemma 5.2 With the notations just established, there holds

$$
\langle\sigma\rangle_{B, Q} \simeq \int_{0}^{\infty} D_{Q}(\lambda) \beta\left(D_{Q}(\lambda)^{-1}\right) d \lambda .
$$

Proof. Both sides scale as a norm. So, assuming that $\|\sigma\|_{B, Q}=1$, we have

$$
\begin{aligned}
1=\frac{1}{|Q|} \int_{Q} A(\sigma(x)) d x & =\int_{0}^{\infty} A(t) d D_{Q}(t) \\
& \simeq \int_{0}^{\infty} \beta(t) \cdot D_{Q}(t) d t
\end{aligned}
$$

by integration by parts. Now, by Chebyshceff, $D_{Q}(t) \leq t^{-1}$, hence

$$
1 \lesssim \int_{0}^{\infty} \beta\left(D_{Q}(t)^{-1}\right) \cdot D_{Q}(t) d t .
$$

For the reverse inequality, we need to see that $\int^{\infty} \beta\left(D_{Q}(t)^{-1}\right)$. $D_{Q}(t) d t \lesssim 1$. And, so we need only be concerned with those $t$ for which $\beta\left(D_{Q}(t)^{-1}\right)>C \beta(t)$. But, for appropriate $C$, this would imply that $D_{Q}(t) \leq \min \left\{1, t^{-2}\right\}$, so the proof is finished.

Definition 5.3 ([17, Definition 2]) A function $\mathrm{f}$ is weakly concave on an interval $I$, if there is a constant $C \geq 1$ so that for any numbers $x_{1}, \ldots, x_{n} \in I$ in its domain, and $\lambda_{1}, \ldots, \lambda_{n} \geq 0$, with $\sum_{j=1}^{n} \lambda_{j}=1$, there holds

$$
f\left(\sum_{j=1}^{n} \lambda_{j} x_{n}\right) \geq C \sum_{j=1}^{n} \lambda_{j} f\left(x_{n}\right) .
$$

Theorem F ([17, Theorem 5.3]) Write $B_{0}(t)=t \beta_{0}(t)$, and assume $B_{0}(t) \lesssim B(t) \theta(\beta(t))$, where $\theta(t)$ is decreasing with $t \rightarrow t \theta(t)$ weakly concave on $(0, \infty)$. Then, 


$$
\langle\sigma\rangle_{B_{0}, Q} \leq C\langle\sigma\rangle_{B, Q} \theta\left(\frac{\langle\sigma\rangle_{B, Q}}{\langle\sigma\rangle_{Q}}\right)
$$

The proof of the theorem relies upon Lemma 5.2, and a Jensen inequality for weakly concave functions. (The constant in the Jensen inequality depends upon the constant in weak convexity.)

Proof. We can assume that $\langle\sigma\rangle_{Q}=1$, so that $\int_{0}^{\infty} D_{Q}(\lambda) d \lambda=1$. There holds, by Lemma 5.2,

$$
\begin{aligned}
\langle\sigma\rangle_{B_{0}, Q} & \simeq \int_{0}^{\infty} D_{Q}(\lambda) \beta_{0}\left(D_{Q}(\lambda)\right) d \lambda \\
& \lesssim \int_{0}^{\infty} D_{Q}(\lambda) \beta\left(D_{Q}(\lambda)\right) \theta\left(\beta\left(D_{Q}(\lambda)\right)\right) d \lambda \\
& =\int_{0}^{\infty} D_{Q}(\lambda) \tilde{\theta}\left(\beta\left(D_{Q}(\lambda)\right) d \lambda\right. \\
& \left.\lesssim \tilde{\theta}\left(\int_{0}^{\infty} D_{Q}(\lambda) \beta\left(D_{Q}(\lambda)\right) d \lambda\right) t \theta(t)\right) \\
& =\int_{0}^{\infty} D_{Q}(\lambda) \beta\left(D_{Q}(\lambda)\right) d \lambda \times \theta\left(\int_{0}^{\infty} D_{Q}(\lambda) \beta\left(D_{Q}(\lambda)\right) d \lambda\right) \\
& \simeq\langle\sigma\rangle_{B, Q} \theta\left(\langle\sigma\rangle_{B, Q}\right) .
\end{aligned}
$$

The first inequality is just hypothesis, and the second inequality is the Jensen inequaltiy which is an immediate corollary to weak concavity. Finally, the expression is rewritten. This proves the Theorem.

Proof of Proposition 5.1. In the first case, we have $[\sigma, w]_{\overline{L_{p^{\prime}, \eta}}, p^{\prime}}<\infty$. Set

$$
B(u):=u(\log u)^{p^{\prime}-1+\eta}, \quad \theta(u):=(1+u)^{-\eta / 2} .
$$

Then, set $B_{0}(u):=B(u) \theta(\log u)$, and $\overline{A_{0}}:=B_{0}\left(t^{p}\right)$. From the definitions, $A_{0}(t)=L_{p^{\prime}, \eta / 2}$, and

$$
\left\langle\sigma^{1 / p^{\prime}}\right\rangle_{A_{0}, Q}^{p^{\prime}}:=\inf \left\{\lambda^{p^{\prime}}>0: \frac{1}{|Q|} \int_{Q} B_{0}\left(\sigma / \lambda^{p^{\prime}}\right) \leq 1\right\}=\langle\sigma\rangle_{B_{0}, Q}
$$




$$
\lesssim\langle\sigma\rangle_{B, Q} \theta\left(\frac{\langle\sigma\rangle_{B, Q}}{\sigma(Q)}\right)
$$

The last line follows from the Theorem $\mathrm{F}$, and the observation that $t \rightarrow t \theta(t)$ is weakly concave.

With $\varepsilon_{p}(t)^{p^{\prime}}:=\theta(t)^{-1}$, it follows from our assumptions that $A_{0} \in B_{p}$, and moreover that

$$
\begin{aligned}
\varepsilon_{p}(t)^{p^{\prime}}\left\langle\sigma^{1 / p^{\prime}}\right\rangle \frac{p^{\prime}}{A_{0}, Q}\langle w\rangle^{p^{\prime} / p} & \lesssim\langle\sigma\rangle_{B, Q}\langle w\rangle^{p^{\prime} / p} \\
& =\left\langle\sigma^{1 / p^{\prime}}\right\rangle \frac{p^{\prime}}{A, Q}\langle w\rangle^{p^{\prime} / p} \leq[\sigma, w] \frac{p^{\prime}}{L_{p^{\prime}, \eta}, p^{\prime}}
\end{aligned}
$$

And this completes this case, since the integral condition (5.1) becomes, after a change of variables $e^{u}=t$,

$$
\int^{\infty} \varepsilon_{p}(t)^{-p^{\prime}} \frac{d t}{t}=\int^{\infty} \mathrm{e}^{-\eta u / 2} d u<\infty .
$$

In this case, the assumption is $[\sigma, w]_{\Lambda_{p^{\prime}, 1+\eta}, p^{\prime}}<\infty$. Recall that in this case we have

$$
\overline{\Lambda_{p^{\prime}, 1+\eta}}(t) \simeq t^{p^{\prime}}\left(\log t^{p^{\prime}}\right)^{1 /(p-1)}\left(\log \log t^{p^{\prime}}\right)^{1 /(p-1)+1+\eta} .
$$

Set

$B(u):=u(\log u)^{1 /(p-1)}(\log \log u)^{1 /(p-1)+\eta / 2}, \quad \theta(u):=(\log u)^{-1-\eta^{\prime} / 2}$.

Then, $B_{0}(u):=B(u) \theta(u)$, and $\overline{A_{0}}(t):=B\left(t^{p}\right)$. Then, $A_{0} \in B_{p}$. The function $t \mapsto t \theta(t)$ is weakly concave. (One can consult [17, Section 5.2.2], or alternatively, check that $\theta$ is concave on $\left[C_{0}, \infty\right)$, for sufficiently large $C_{0}$.) Thus, (5.3) continues to hold in this case.

With $\varepsilon_{p}(t)^{p^{\prime}}:=\theta(t)^{-1}$, the inequality (5.4) will continue to hold, with obvious changes. And the integral condition (5.1) holds, since with the same exponential change of variables,

$$
\int^{\infty} \varepsilon_{p}(t)^{-p^{\prime}} \frac{d t}{t}=\int^{\infty} \frac{d u}{u^{1+\eta / 2}}<\infty .
$$




\section{References}

[ 1 ] Anderson T. C., Cruz-Uribe D. and Moen K., Logarithmic bump conditions for Calderón-Zygmund operators on spaces of homogeneous type. Publ. Mat. 59 (2015), 17-43.

[2] Chang S.-Y. A., Wilson J. M. and Wolff T. H., Some weighted norm inequalities concerning the Schrödinger operators. Comment. Math. Helv. 60 (1985), 217-246.

[ 3 ] Cruz-Uribe D. V., Martell J. M. and Pérez C., Sharp two-weight inequalities for singular integrals, with applications to the Hilbert transform and the Sarason conjecture. Adv. Math., 216 (2007), 647-676.

[ 4 ] Cruz-Uribe D. V., Martell J. M. and Pérez C., Weights, extrapolation and the theory of Rubio de Francia. Operator Theory: Advances and Applications, 215, Birkhäuser/Springer Basel AG, Basel, 2011.

[ 5 ] Cruz-Uribe D., Martell J. M. and Pérez C., Sharp weighted estimates for classical operators. Adv. Math., 229 (2012), 408-441.

[6 ] Cruz-Uribe D. and Pérez C., Two-weight, weak-type norm inequalities for fractional integrals, Calderón-Zygmund operators and commutators. Indiana Univ. Math. J., 49 (2000), 697-721.

[ 7 ] Cruz-Uribe D. and Pérez C., Sharp two-weight, weak-type norm inequalities for singular integral operators. Math. Res. Lett., 6 (1999), 417-427.

[ 8 ] Cruz-Uribe D., Reznikov A. and Volberg A., Logarithmic bump conditions and the two-weight boundedness of Calderón-Zygmund operators. Adv. Math., 255 (2014), 706-729, DOI 10.1016/j.aim.2014.01.016, MR3167497.

[ 9 ] Hunt R., Muckenhoupt B. and Wheeden R., Weighted norm inequalities for the conjugate function and Hilbert transform. Trans. Amer. Math. Soc., 176 (1973), 227-251.

[10] Hytönen T. P., The sharp weighted bound for general Calderón-Zygmund operators. Ann. of Math. (2), 175 (2012), 1473-1506.

[11] Hytönen T. P., The $A_{2}$ theorem: remarks and complements. Harmonic analysis and partial differential equations, 91-106, Contemp. Math., 612, Amer. Math. Soc., Providence, RI, 2014.

[12] Lacey M. T., Sawyer E. T. and Uriarte-Tuero I., Two Weight Inequalities for Discrete Positive Operators, Submitted (2009), available at http:// arxiv.org/abs/http://www.arxiv.org/abs/0911.3437.

[13] Lacey M. T., Sawyer E. T., Shen C.-Y. and Uriarte-Tuero I., Two-weight inequality for the Hilbert transform: a real variable characterization, I. Duke Math. J., 163 (2014), 2795-2820.

[14] Lerner A. K., On an estimate of Calderón-Zygmund operators by dyadic positive operators. J. Anal. Math., 121 (2013), 141-161, DOI 10.1007/ 
s11854-013-0030-1, MR3127380.

[15] Lerner A. K., A simple proof of the $A_{2}$ conjecture, Int. Math. Res. Not. IMRN, 14 (2013), 3159-3170, MR3085756.

[16] Nazarov F., Reznikov A., Treil S. and Volberg A., A Bellman function proof of the $L^{2}$ bump conjecture. J. Anal. Math., 121 (2013), 255-277, DOI 10.1007/s11854-013-0035-9, MR3127385.

[17] Nazarov F., Reznikov A. and Volberg A., Bellman approach to the one-sided bumping for weighted estimates of Calderón-Zygmund operators, (2011), available at http://arxiv.org/abs/1306.2653.

[18] Neugebauer C. J., Inserting $A_{p}$-weights. Proc. Amer. Math. Soc., 87 (1983), 644-648.

[19] Pérez C., Weighted norm inequalities for singular integral operators. J. London Math. Soc. (2), 49 (1994), 296-308.

[20] Pérez C., Two weighted inequalities for potential and fractional type maximal operators. Indiana Univ. Math. J., 43 (1994), 663-683.

[21] Sarason D., Products of Toeplitz operators, Linear and complex analysis. Problem book 3. Part I (V. P. Havin and N. K. Nikolski, eds.), Lecture Notes in Mathematics, 1573, Springer-Verlag, Berlin, 1994, pp. 318-319.

[22] Sawyer E. T., A characterization of a two-weight norm inequality for maximal operators. Studia Math., 75 (1982), 1-11.

[23] Sawyer E. T., A characterization of two weight norm inequalities for fractional and Poisson integrals. Trans. Amer. Math. Soc., 308 (1988), 533-545.

[24] Treil S., Volberg A. and Zheng D., Hilbert transform, Toeplitz operators and Hankel operators, and invariant $A_{\infty}$ weights. Rev. Mat. Iberoamericana, 13 (1997), 319-360.

[25] Zheng D., The distribution function inequality and products of Toeplitz operators and Hankel operators. J. Funct. Anal., 138 (1996), 477-501.

School of Mathematics

Georgia Institute of Technology

Atlanta GA 30332, USA

E-mail: lacey@math.gatech.edu 